\title{
Second order approximations for kinetic and potential energies in Maxwell's wave equations
}

\author{
J.A. Ferreira ${ }^{(1)}$, D. Jordão ${ }^{(2)}$, L. Pinto ${ }^{(1)}$ \\ (1) CMUC, Department of Mathematics, University of Coimbra, Portugal \\ (2) Department of Mathematics, University of Coimbra, Portugal \\ ferreira@mat.uc.pt,daniela.jordao@hotmail.com, luisp@mat.uc.pt
}

March 14, 2017

\begin{abstract}
In this paper we propose a numerical scheme for wave type equations with damping and space variable coefficients. Relevant equations of this kind arise for instance in the context of Maxwell's equations, namely, the electric potential equation and the electric field equation. The main motivation to study such class of equations is the crucial role played by the electric potential or the electric field in enhanced drug delivery applications. Our numerical method is based on piecewise linear finite element approximation and it can be regarded as a finite difference method based on non-uniform partitions of the spatial domain. We show that the proposed method leads to second order convergence, in time and space, for the kinetic and potential energies with respect to a discrete $L^{2}$-norm.
\end{abstract}

keywords: enhanced drug delivery, Maxwell's equations, finite element method, finite difference method, supercloseness, supraconvergence.

\section{Introduction}

We study in what follows a discretization in time and space of the following wave equation

$$
a \frac{\partial^{2} u}{\partial t^{2}}+b \frac{\partial u}{\partial t}=\nabla \cdot(D \nabla u)+f \text { in } \Omega \times(0, T],
$$

with the initial conditions

$$
\left\{\begin{array}{l}
\frac{\partial u}{\partial t}(x, y, 0)=\phi_{1}(x, y) \\
u(x, y, 0)=\phi_{0}(x, y),(x, y) \in \Omega
\end{array}\right.
$$

and homogeneous Dirichlet boundary condition

$$
u=0 \text { in } \partial \Omega \times(0, T] .
$$

By simplicity we assume that $\Omega=(0,1) \times(0,1)$ and $u: \bar{\Omega} \times[0, T] \rightarrow \mathbb{R}$. In equation (1), the coefficient functions are $x$ and $y$ dependent and $a \geq a_{0}>0$ and $b \geq b_{0} \geq 0$ in $\bar{\Omega}$, and $D$ represents a diagonal matrix with positive diagonal entries $d_{i}, i=1,2$, that have in $\bar{\Omega}$ a positive lower bound $d_{0}$. 
Equation (1), a wave equation with a damping factor, has as a particular case the potential equation that arises from the following wave equation

$$
\mu \epsilon \sigma \frac{\partial^{2} V}{\partial t^{2}}+\mu \sigma^{2} \frac{\partial V}{\partial t}=\nabla \cdot(\sigma \nabla V)-\frac{\partial \rho}{\partial t}
$$

where $V$ denotes the scalar potential, $\epsilon$ the electric permittivity, $\mu$ the magnetic permeability, $\sigma$ the conductivity of the medium, and $\rho$ the charge density of the current (see for instance [25]).

We remark that the results that we present in this paper can be easily extended to the initial boundary value problem (IBVP) (1)-(3) when $u: \bar{\Omega} \times[0, T] \rightarrow \mathbb{R}^{n}$. In this case, for $n=3$, equation (1) has as a particular case the electric field equation

$$
\mu \epsilon \frac{\partial^{2} E}{\partial t^{2}}+\mu \sigma \frac{\partial E}{\partial t}=\Delta E-\nabla\left(\frac{\rho}{\epsilon}\right)
$$

where $E$ represents the electric field, $\Delta E$ the vector with components $\Delta E_{i}$ and $\nabla\left(\frac{\rho}{\epsilon}\right)$ the gradient of $\frac{\rho}{\epsilon}$ (see for instance $[25]$ ).

Our main motivation for this paper is the coupling between drug transport and electric current, which is used in several medical applications like transdermal drug delivery $([21,22$, $28,35])$, cancer treatment $([10,38])$ or ophthalmic applications $([32])$. In all these applications, the drug transport is enhanced by the applied electric current. The drug mass flux is described by the Nernst-Planck equation and it is given by three main contributions: passive transport due to drug diffusion, electric enhanced drug transport that depends on the electric potential gradient or electric field, and electroosmotic transport due to fluid flow $([21,33,35])$.

In the mathematical description of the drug time-space evolution, the authors usually assume that the potential is described by a Poisson equation when iontophoretic or electroporation protocols are applied. Without being exhaustive we refer to $[6,14,20]$. However, to obtain an accurate description of the drug evolution in a more general setting, it is necessary to construct an accurate approximation for the electric potential $V$ defined by (4) or electric field defined by (5). It is desirable to compute a second order approximation for the gradient of the potential with respect to a discrete $L^{2}$-norm, that is, a second order approximation for the potential with respect to a discrete $H^{1}$-norm. In what concerns the electric field, the corresponding scheme leads to a second order approximation with respect to a $\left[H^{1}\right]^{3}$-discrete norm.

The method that we propose is obtained considering the well known MOL approach ([36]): a spatial discretization that leads to a semi-discrete approximation (continuous in time) followed by a time integration. The spatial discretization is defined considering a piecewise linear finite element method combined with particular integration rules that lead to a fully discrete in space scheme. It should be remarked that the constructed fully discrete scheme can be seen as a finite difference method.

The classical convergence analysis of the semi-discrete approximation using the finite difference language is based on the concept of truncation error. Although the truncation error is only of first order with respect to the norm $\|\cdot\|_{\infty}$, when general non-uniform grids are considered, using our approach we prove that the finite difference approximation for the solution of the IBVP (1)-(3) is second order convergent with respect to a discrete $H^{1}$-norm provided that $u^{\prime}(t), u(t) \in C^{4}(\bar{\Omega}), t \in(0, T]$. This means that the corresponding numerical gradient is second order convergent with respect to a discrete $L^{2}$-norm.

Furthermore, to reduce the smoothness assumptions on the solution of the IBVP (1)-(3), we consider the approach introduced in [4] for one dimensional problems and in [16] for two dimensional elliptic equations and consider later in different contexts: in $[5,17,19]$ for non-Fickian 
diffusion problems, and in [18] for diffusion problems in porous media. Avoiding the analytical difficulties that arise from the application of this technique we prove the same convergence result provided that $u^{\prime}(t), u(t) \in H^{3}(\Omega), t \in(0, T]$.

As observed before, the semi-discrete finite difference approximation is also a fully discrete in space piecewise linear finite element approximation. In this context the obtained result is unexpected and is usually referred as a superclose result ([37]). There exist many papers about numerical methods for wave type equations, including finite differences $([1,12])$, finite elements $([3,15])$, mixed finite elements $([11,13,23])$, and discontinuous Galerkin $([24,30,34])$. On the other hand, only a few works have been dedicated to superclose (or superconvergent) estimates, some examples are $[2,9,26,27,29,31]$.

The paper is organized as follows. In Section 2 we introduce the definitions and notations used in this work and formulate our fully discrete in space method. The convergence analysis of the semi-discrete approximation for the solution of the IBVP (1)-(3) when the solution $u$ is smooth, that is, $u^{\prime}(t), u(t) \in C^{4}(\bar{\Omega})$, is presented in Section 3. Section 4 is focused on the extension of this analysis to the non-smooth case, that is, when $u^{\prime}(t), u(t) \in H^{3}(\Omega)$. The fully discrete in time and space method is studied in Section 5 and numerical results illustrating the theoretical results established in the previous sections are presented in Section 6. Finally, in Section 7 , we draw some conclusions and future work directions.

\section{$2 \quad$ Fully discrete approximation in space}

By $L^{2}(\Omega), H_{0}^{1}(\Omega)$ we denote the usual Sobolev spaces equipped with the norms $\|\cdot\|_{0},\|\cdot\|_{1}$ induced by the corresponding inner products $(.,$.$) and (., .)_{1}$. The usual inner product in $\left(L^{2}(\Omega)\right)^{2}$ is represented by $((.,)$.$) . If v: \bar{\Omega} \times[0, T] \rightarrow \mathbb{R}$, then $v(t): \bar{\Omega} \rightarrow \mathbb{R}$ with $v(t)(x)=v(x, t), x \in \bar{\Omega}$.

Let us consider the following variational problem: find $u(t) \in H_{0}^{1}(\Omega)$ such that

$$
\left(a u^{\prime \prime}(t), w\right)+\left(b u^{\prime}(t), w\right)=-((D \nabla u, \nabla w))+(f(t), w), t \in(0, T],
$$

for $w \in H_{0}^{1}(\Omega)$, and

$$
\left\{\begin{array}{l}
u^{\prime}(0)=\phi_{1} \\
u(0)=\phi_{0}
\end{array}\right.
$$

In $\bar{\Omega}$ we introduce a non-uniform rectangular grid defined by $H=(h, k)$ with $h=\left(h_{1}, \ldots, h_{N}\right)$, $h_{i}>0, i=1, \ldots, N, \sum_{i=1}^{N} h_{i}=1$, and $k=\left(k_{1}, \ldots, k_{M}\right), k_{j}>0, j=1, \ldots, M, \sum_{j=1}^{M} k_{j}=1$. Let $\left\{x_{i}\right\}$ and $\left\{y_{j}\right\}$ be the non-uniform grids induced by $h$ and $k$ in $[0,1]$ with $x_{i}-x_{i-1}=h_{i}, y_{j}-y_{j-1}=k_{j}$. By $\bar{\Omega}_{H}$ we represent the rectangular grid introduced in $\bar{\Omega}$ that depends on $H$ and let $\Omega_{H}$ and $\partial \Omega_{H}$ be defined by $\Omega_{H}=\Omega \cap \bar{\Omega}_{H}, \partial \Omega_{H}=\partial \Omega \cap \bar{\Omega}_{H}$.

Let $H_{\text {max }}=\max \left\{h_{i}, k_{j} ; i=1, \cdots N ; j=1, \cdots, M\right\}$. By $\Lambda$ we denote a sequence of vectors $H=(h, k)$ such that $H_{\max } \rightarrow 0$. Let $W_{H}$ be the space of grid functions defined in $\bar{\Omega}_{H}$ and by $W_{H, 0}$ we denote the subspace of $W_{H}$ of grid functions null on $\partial \Omega_{H}$. Let $\mathcal{T}_{H}$ be a triangulation of $\bar{\Omega}$ using the set $\bar{\Omega}_{H}$ as vertices. We denote by diam $\Delta$ the diameter of the triangle $\Delta \in \mathcal{T}_{H}$. By $P_{H} v_{H}$ we denote the continuous piecewise linear interpolant of $v_{H}$ with respect to $\mathcal{T}_{H}$.

We consider now the following piecewise linear finite element problem: find $u_{H}(t) \in W_{H, 0}$ such that

$$
\left(a P_{H} u_{H}^{\prime \prime}(t), P_{H} w_{H}\right)+\left(b P_{H} u_{H}^{\prime}(t), P_{H} w_{H}\right)=-\left(\left(D \nabla P_{H} u_{H}(t), \nabla P_{H} w_{H}\right)\right)+\left(f(t), P_{H} w_{H}\right),
$$


for $t \in(0, T]$ and

$$
\left\{\begin{array}{l}
\left(P_{H} u_{H}^{\prime}(0), P_{H} w_{H}\right)=\left(P_{H} R_{H} \phi_{1}, P_{H} w_{H}\right) \\
\left(P_{H} u_{H}(0), P_{H} w_{H}\right)=\left(P_{H} R_{H} \phi_{0}, P_{H} w_{H}\right),
\end{array}\right.
$$

for $w_{H} \in W_{H, 0}$. In (7), $R_{H}: C(\bar{\Omega}) \rightarrow W_{H}$ denotes the restriction operator, where $C(\bar{\Omega})$ represents the space of continuous functions in $\bar{\Omega}$.

A fully discrete in space approximation is introduced now. In $W_{H, 0}$ we define the inner product

$$
\left(v_{H}, w_{H}\right)_{H}=\sum_{\left(x_{i}, y_{j}\right) \in \bar{\Omega}_{H}}\left|\square_{i, j}\right| v_{H}\left(x_{i}, y_{j}\right) w_{H}\left(x_{i}, y_{j}\right), w_{H}, v_{H} \in W_{H, 0},
$$

where $\square_{i, j}=\left(x_{i-1 / 2}, x_{i+1 / 2}\right) \times\left(y_{j-1 / 2}, y_{j+1 / 2}\right) \cap \Omega,\left|\square_{i, j}\right|$ denotes the area of $\square_{i, j}$, and $x_{i+1 / 2}=$ $x_{i}+\frac{h_{i+1}}{2}, x_{i-1 / 2}=x_{i}-\frac{h_{i}}{2}, h_{i+1 / 2}=x_{i+1 / 2}-x_{i-1 / 2}$ being $y_{j \pm 1 / 2}$ and $k_{j+1 / 2}$ defined analogously. Let $\|\cdot\|_{H}$ be the corresponding norm.

For $v_{H}=\left(v_{1, H}, v_{2, H}\right), w_{H}=\left(w_{1, H}, w_{2, H}\right)$, and $v_{\ell, H}, w_{\ell, H} \in W_{H}$, for $\ell=1,2$, we use the notation

$$
\left(\left(v_{H}, w_{H}\right)\right)_{H}=\left(v_{1, H}, w_{1, H}\right)_{H, x}+\left(v_{2, H}, w_{2, H}\right)_{H, y},
$$

where

$$
\left(v_{1, H}, w_{1, H}\right)_{H, x}=\sum_{i=1}^{N} \sum_{j=1}^{M-1} h_{i} k_{j+1 / 2} v_{1, H}\left(x_{i}, y_{j}\right) w_{1, H}\left(x_{i}, y_{j}\right),
$$

being $\left(v_{2, H}, w_{2, H}\right)_{H, y}$ defined analogously.

Let $D_{-x}$ and $D_{-y}$ be the first order backward finite difference operators with respect to the variables $x$ and $y$, respectively, and let $\nabla_{H}$ be the discrete version of the gradient operator $\nabla$ defined by $\nabla_{H} u_{H}=\left(D_{-x} u_{H}, D_{-y} u_{H}\right)$. By $D_{H}$ we denote the diagonal matrix with $d_{1, H}\left(x_{i}, y_{j}\right)=$ $d_{1}\left(x_{i-1 / 2}, y_{j}\right)$ and $d_{2, H}\left(x_{i}, y_{j}\right)=d_{2}\left(x_{i}, y_{j-1 / 2}\right)$, for $\left(x_{i}, y_{j}\right) \in \Omega_{H}$.

The initial value problem (6), (7) is replaced by the following fully discrete in space finite element problem: find $u_{H}(t) \in W_{H, 0}$ such that

$$
\left(a_{H} u_{H}^{\prime \prime}(t), w_{H}\right)_{H}+\left(b_{H} u_{H}^{\prime}(t), w_{H}\right)_{H}=-\left(\left(D_{H} \nabla_{H} u_{H}(t), \nabla_{H} w_{H}\right)\right)_{H}+\left(f_{H}(t), w_{H}\right)_{H},
$$

for $t \in(0, T], w_{H} \in W_{H, 0}$, and

$$
\left\{\begin{array}{l}
u_{H}^{\prime}(0)=R_{H} \phi_{1} \\
u_{H}(0)=R_{H} \phi_{0}
\end{array}\right.
$$

In (8), $a_{H}=R_{H} a, b_{H}=R_{H} b$, and

$$
f_{H}(t)\left(x_{i}, y_{j}\right)=\frac{1}{\left|\square_{i, j}\right|} \int_{\square_{i, j}} f(x, y, t) d x d y .
$$

We observe that the fully discrete in space finite element problem can be rewritten as a finite difference problem. In order to define such finite difference problem, we introduce the finite difference operator $\nabla_{H}^{*}=\left(D_{x}^{*}, D_{y}^{*}\right)$ where

$$
D_{x}^{*} v_{H}\left(x_{i}, y_{j}\right)=\frac{v_{H}\left(x_{i+1}, y_{j}\right)-v_{H}\left(x_{i}, y_{j}\right)}{h_{i+1 / 2}},
$$

and $D_{y}^{*}$ is defined analogously.

Then, from (8) we obtain

$$
a_{H} u_{H}^{\prime \prime}(t)+b_{H} u_{H}^{\prime}(t)=\nabla_{H}^{*} \cdot\left(D_{H} \nabla_{H} u_{H}(t)\right)+f_{H}(t) \text { in } \Omega_{H}, t \in(0, T],
$$


which is coupled with the boundary condition

$$
u_{H}(t)=0 \text { on } \partial \Omega_{H},
$$

and the initial conditions (9).

In the next section we study the convergence properties of the fully discrete approximation $u_{H}(t)$ defined by (11), (9), and (12) or equivalently by (8), (9), and (12). The analysis technique depends on the smoothness of the solution $u$ of the corresponding IBVP (1)-(3).

\section{Convergence analysis: smooth case}

Traditionally, the convergence analysis of a semi-discrete approximation $u_{H}(t)$ is based on the truncation error $T_{H}(t)$ associated with the spatial discretization. Assume for simplicity, but without loss of generality, that $d_{i}=1, i=1,2$. Under this assumption, we have

$$
\begin{aligned}
T_{H}(t)=-( & \left.h_{i+1}-h_{i}\right)\left(\frac{1}{3} R_{H} \frac{\partial^{3} u}{\partial x^{3}}(t)+\frac{1}{4} R_{H} \frac{\partial f}{\partial x}(t)\right) \\
& -\left(k_{j+1}-k_{j}\right)\left(\frac{1}{3} R_{H} \frac{\partial^{3} u}{\partial y^{3}}(t)+\frac{1}{4} R_{H} \frac{\partial f}{\partial y}(t)\right)+O\left(H_{\max }^{2}\right),
\end{aligned}
$$

provided that $u(t) \in C^{4}(\bar{\Omega}), f \in C^{2}(\bar{\Omega})$. In $T_{H}(t)$, the term $O\left(H_{\max }^{2}\right)$ represents a term such that there exists a positive constant $C_{T}$ satisfying

$$
\left|O\left(H_{\max }^{2}\right)\right| \leq C_{T} H_{\max }^{2}\left(\|u(t)\|_{C^{4}}+\|f(t)\|_{C^{2}}\right)
$$

where $\|\cdot\|_{C^{m}}$ denotes the usual norm in $C^{m}(\bar{\Omega}), m \in \mathbb{N}_{0}$.

Let $e_{H}(t)=R_{H} u(t)-u_{H}(t)$ be the spatial discretization error induced by the numerical scheme. The spatial and the correspondent truncation errors satisfy the following equation

$$
\left(a_{H} e_{H}^{\prime \prime}(t), w_{H}\right)_{H}+\left(b_{H} e_{H}^{\prime}(t), w_{H}\right)_{H}=-\left(\left(D_{H} \nabla_{H} e_{H}(t), \nabla_{H} w_{H}\right)\right)_{H}+\left(T_{H}(t), w_{H}\right)_{H},
$$

for $t \in(0, T], w_{H} \in W_{H, 0}$, and

$$
\left\{\begin{array}{l}
e_{H}^{\prime}(0)=0 \\
e_{H}(0)=0
\end{array}\right.
$$

In the next result we establish an upper bound for the kinetic and potential energies of $e_{H}(t)$ where the potential energy is defined by considering the semi-norm

$$
\left\|\nabla_{H} w_{H}\right\|_{H}=\left(\left(\left(\nabla_{H} w_{H}, \nabla_{H} w_{H}\right)\right)_{H}\right)^{1 / 2}, w_{H} \in W_{H} .
$$

Here, we denote by $\|\cdot\|_{C^{m}\left(C^{p}\right)}$ the usual norm in $C^{m}\left(0, T, C^{p}(\bar{\Omega})\right), m, p \in \mathbb{N}_{0}$. We observe that this result is established under too restrictive smoothness assumptions that will be weakened later. We also remark that weaker conditions were considered for first order hyperbolic problems in [39].

Theorem 1. If the solution $u$ of the IBVP (1)-(3) is in $C^{1}\left(0, T, C^{4}(\bar{\Omega})\right) \cap C^{2}(0, T, C(\bar{\Omega}))$ and $f \in C^{1}\left(0, T, C^{2}(\bar{\Omega})\right)$, then there exist positive constants $C_{1}, C_{2}$, independent of $u, f, H$, and $T$, such that for $H \in \Lambda$ with $H_{\max }$ small enough

$$
\begin{aligned}
\left\|e_{H}^{\prime}(t)\right\|_{H}^{2}+\int_{0}^{t}\left\|e_{H}^{\prime}(s)\right\|_{H}^{2} d s+\left\|e_{H}(t)\right\|_{H}^{2}+\left\|\nabla_{H} e_{H}(t)\right\|_{H}^{2} \\
\leq C_{1} H_{\text {max }}^{4} e^{C_{2} t}\left(\|u\|_{C^{1}\left(C^{4}\right)}^{2}+\|f\|_{C^{1}\left(C^{2}\right)}^{2}\right), t \in[0, T] .
\end{aligned}
$$


Proof: From (13) we get

$$
\left(a_{H} e_{H}^{\prime \prime}(t), e_{H}^{\prime}(t)\right)_{H}+b_{0}\left\|e_{H}^{\prime}(t)\right\|_{H}^{2} \leq-\left(\left(D_{H} \nabla_{H} e_{H}(t), \nabla_{H} e_{H}^{\prime}(t)\right)\right)_{H}+\left(T_{H}(t), e_{H}^{\prime}(t)\right)_{H}, t \in(0, T]
$$

that leads to

$$
\frac{d}{d t}\left(\left\|\sqrt{a_{H}} e_{H}^{\prime}(t)\right\|_{H}^{2}+2 b_{0} \int_{0}^{t}\left\|e_{H}^{\prime}(s)\right\|_{H}^{2} d s+\left\|\sqrt{D_{H}} \nabla_{H} e_{H}(t)\right\|_{H}^{2}\right) \leq 2\left(T_{H}(t), e_{H}^{\prime}(t)\right)_{H},
$$

for $t \in(0, T]$. In (15), $\sqrt{D_{H}}$ is the diagonal matrix whose entries are given by $\sqrt{d_{i}}, i=$ 1,2 . The main difficulty in the construction of a convenient upper bound for $\left\|\sqrt{a_{H}} e_{H}^{\prime}(t)\right\|_{H}^{2}+$ $2 b_{0} \int_{0}^{t}\left\|e_{H}^{\prime}(s)\right\|_{H}^{2} d s+\left\|\sqrt{D_{H}} \nabla_{H} e_{H}(t)\right\|^{2}$ is related with the term $\left(T_{H}(t), e_{H}^{\prime}(t)\right)_{H}$. We observe that

$$
\left(T_{H}(t), e_{H}^{\prime}(t)\right)_{H}=\frac{d}{d t}\left(T_{H}(t), e_{H}(t)\right)_{H}-\left(T_{H}^{\prime}(t), e_{H}(t)\right)_{H},
$$

where

$$
\begin{aligned}
& T_{H}^{\prime}(t)=-(\left.h_{i+1}-h_{i}\right)\left(\frac{1}{3} R_{H} \frac{\partial^{4} u}{\partial t \partial x^{3}}(t)+\frac{1}{4} R_{H} \frac{\partial^{2} f}{\partial t \partial x}(t)\right) \\
&-\left(k_{j+1}-k_{j}\right)\left(\frac{1}{3} R_{H} \frac{\partial^{4} u}{\partial t \partial y^{3}}(t)+\frac{1}{4} R_{H} \frac{\partial^{2} f}{\partial t \partial y}(t)\right)+O\left(H_{\text {max }}^{2}\right),
\end{aligned}
$$

with $\left|O\left(H_{\max }^{2}\right)\right| \leq C_{T} H_{\max }^{2}\left(\left\|u^{\prime}(t)\right\|_{C^{4}}+\left\|f^{\prime}(t)\right\|_{C^{2}}\right)$.

From (15) and (16) we get

$$
\begin{aligned}
\left\|\sqrt{a_{H}} e_{H}^{\prime}(t)\right\|_{H}^{2}+2 b_{0} \int_{0}^{t}\left\|e_{H}^{\prime}(s)\right\|_{H}^{2} d s+\left\|\sqrt{D_{H}} \nabla_{H} e_{H}(t)\right\|_{H}^{2} \leq 2\left(T_{H}(t), e_{H}(t)\right)_{H} \\
-2 \int_{0}^{t}\left(T_{H}^{\prime}(s), e_{H}(s)\right)_{H} d s, t \in(0, T] .
\end{aligned}
$$

To obtain upper bounds for the terms $\left(T_{H}(t), e_{H}(t)\right)_{H},\left(T_{H}^{\prime}(s), e_{H}(s)\right)_{H}$ we consider the generic term

$$
T_{G, x}(t)=\sum_{i=1}^{N-1} \sum_{j=1}^{M-1} h_{i+1 / 2} k_{j+1 / 2}\left(h_{i+1}-h_{i}\right) v\left(x_{i}, y_{j}, t\right) e_{H}\left(x_{i}, y_{j}, t\right) .
$$

We have successively

$$
\begin{aligned}
T_{G, x}(t)= & \frac{1}{2} \sum_{i=1}^{N-1} \sum_{j=1}^{M-1} k_{j+1 / 2}\left(h_{i+1}^{2}-h_{i}^{2}\right)\left(v\left(x_{i}, y_{j}, t\right) e_{H}\left(x_{i}, y_{j}, t\right)\right) \\
= & -\frac{1}{2} \sum_{i=1}^{N} \sum_{j=1}^{M-1} k_{j+1 / 2} h_{i}^{2}\left(v\left(x_{i}, y_{j}, t\right) e_{H}\left(x_{i}, y_{j}, t\right)-v\left(x_{i-1}, y_{j}, t\right) e_{H}\left(x_{i-1}, y_{j}, t\right)\right) \\
= & -\frac{1}{2} \sum_{i=1}^{N} \sum_{j=1}^{M-1} k_{j+1 / 2} h_{i}^{2} \int_{x_{i-1}}^{x_{i}} \frac{\partial v}{\partial x}\left(x, y_{j}, t\right) d x e_{H}\left(x_{i}, y_{j}, t\right) \\
& -\frac{1}{2} \sum_{i=1}^{N} \sum_{j=1}^{M-1} k_{j+1 / 2} h_{i}^{3} v\left(x_{i-1}, y_{j}, t\right) D_{-x} e_{H}\left(x_{i}, y_{j}, t\right) \\
:= & T_{G, x}^{(1)}(t)+T_{G, x}^{(2)}(t) .
\end{aligned}
$$


From this, we can establish the following upper bounds

$$
\left|T_{G, x}^{(1)}(t)\right| \leq \frac{1}{4 \eta_{1}^{2}} H_{\text {max }}^{4}\|v(t)\|_{C^{1}}^{2}+\eta_{1}^{2}\left\|e_{H}(t)\right\|_{H}^{2}
$$

and

$$
\left|T_{G, x}^{(2)}(t)\right| \leq \frac{1}{4 \eta_{2}^{2}} H_{\max }^{4}\|v(t)\|_{C^{0}}^{2}+\eta_{2}^{2}\left\|D_{-x} e_{H}(t)\right\|_{H, x}^{2},
$$

where $\eta_{i}, i=1,2$, are non-zero constants, and consequently

$$
\left|T_{G, x}(t)\right| \leq \frac{1}{4 \eta_{1}^{2}} H_{\max }^{4}\|v(t)\|_{C^{1}}^{2}+\eta_{1}^{2}\left\|e_{H}(t)\right\|_{H}^{2}+\frac{1}{4 \eta_{2}^{2}} H_{\text {max }}^{4}\|v(t)\|_{C^{0}}^{2}+\eta_{2}^{2}\left\|D_{-x} e_{H}(t)\right\|_{H, x}^{2} .
$$

For the correspondent $y$ term

$$
T_{G, y}(t)=\sum_{i=1}^{N-1} \sum_{j=1}^{M-1} h_{i+1 / 2} k_{j+1 / 2}\left(k_{j+1}-k_{j}\right) v\left(x_{i}, y_{j}, t\right) e_{H}\left(x_{i}, y_{j}, t\right)
$$

it can be shown that holds the following

$$
\left|T_{G, y}(t)\right| \leq \frac{1}{4 \eta_{3}^{2}} H_{\max }^{4}\|v(t)\|_{C^{1}}^{2}+\eta_{3}^{2}\left\|e_{H}(t)\right\|_{H}^{2}+\frac{1}{4 \eta_{4}^{2}} H_{\text {max }}^{4}\|v(t)\|_{C^{0}}^{2}+\eta_{4}^{2}\left\|D_{-y} e_{H}(t)\right\|_{H, y}^{2},
$$

where $\eta_{i}, i=3,4$, are non-zero constants.

Taking $\eta_{1}=\eta_{3}$ and $\eta_{2}=\eta_{4}$, we deduce

$$
\left|T_{G, x}(t)\right|+\left|T_{G, y}(t)\right| \leq\left(\frac{1}{2 \eta_{1}^{2}}+\frac{1}{2 \eta_{2}^{2}}\right) H_{m a x}^{4}\|v(t)\|_{C^{1}}^{2}+2 \eta_{1}^{2}\left\|e_{H}(t)\right\|_{H}^{2}+\eta_{2}^{2}\left\|\nabla_{H} e_{H}(t)\right\|_{H}^{2} .
$$

From (18) we conclude for $\left(T_{H}(t), e_{H}(t)\right)$ the upper bound

$$
\begin{array}{r}
\left|\left(T_{H}(t), e_{H}(t)\right)_{H}\right| \leq\left(\frac{1}{2 \xi_{1}^{2}}+\frac{1}{2 \xi_{2}^{2}}\right) H_{\max }^{4}\left(\frac{1}{3}\|u(t)\|_{C^{4}}+\frac{1}{4}\|f(t)\|_{C^{2}}\right)^{2}+3 \xi_{1}^{2}\left\|e_{H}(t)\right\|_{H}^{2} \\
+\xi_{2}^{2}\left\|\nabla_{H} e_{H}(t)\right\|_{H}^{2}+\frac{1}{4 \xi_{1}^{2}} C_{T}^{2} H_{\max }^{4}\left(\|u(t)\|_{C^{4}}+\|f(t)\|_{C^{2}}\right)^{2},
\end{array}
$$

where $\xi_{i}, i=1,2$, are non-zero constants, and for $\left(T_{H}^{\prime}(s), e_{H}(s)\right)$ we get the upper bound

$$
\begin{aligned}
\left|\left(T_{H}^{\prime}(s), e_{H}(s)\right)_{H}\right| \leq\left(\frac{1}{2 \xi_{3}^{2}}\right. & \left.+\frac{1}{2 \xi_{4}^{2}}\right) H_{\max }^{4}\left(\frac{1}{3}\left\|u^{\prime}(s)\right\|_{C^{4}}+\frac{1}{3}\left\|f^{\prime}(s)\right\|_{C^{2}}\right)^{2}+3 \xi_{3}^{2}\left\|e_{H}(s)\right\|_{H}^{2} \\
& +\xi_{4}^{2}\left\|\nabla_{H} e_{H}(s)\right\|_{H}^{2}+\frac{1}{4 \xi_{3}^{2}} C_{T}^{2} H_{\max }^{4}\left(\left\|u^{\prime}(t)\right\|_{C^{4}}+\left\|f^{\prime}(t)\right\|_{C^{2}}\right)^{2}
\end{aligned}
$$

where $\xi_{i}, i=3,4$, are non-zero constants.

Considering (19) and (20) in (17) and using the Poincaré-Friedrichs's inequality

$$
\left\|w_{H}\right\|_{H}^{2} \leq \frac{1}{2}\left\|\nabla_{H} w_{H}\right\|_{H}^{2}, w_{H} \in W_{H, 0},
$$

we obtain

$$
\begin{aligned}
& a_{0}\left\|e_{H}^{\prime}(t)\right\|_{H}^{2}+2 b_{0} \int_{0}^{t}\left\|e_{H}^{\prime}(s)\right\|_{H}^{2} d s+\left(\frac{d_{0}}{2}-6 \xi_{1}^{2}\right)\left\|e_{H}(t)\right\|_{H}^{2}+\left(\frac{d_{0}}{2}-2 \xi_{2}^{2}\right)\left\|\nabla_{H} e_{H}(t)\right\|^{2} \\
& \leq \int_{0}^{t} 2\left(\xi_{4}^{2}\left\|\nabla_{H} e_{H}(s)\right\|_{H}^{2}+3 \xi_{3}^{2}\left\|e_{H}(s)\right\|_{H}^{2}\right) d s+C H_{\max }^{4}\left(R(t)+\int_{0}^{t} R(s) d s\right), t \in(0, T],
\end{aligned}
$$


where $C$ depends on the previous constants $\xi_{i}, i=1,2,3,4$, and $C_{T}$ and $R(\mu)=\|u(\mu)\|_{C^{4}}^{2}+$ $\left\|u^{\prime}(\mu)\right\|_{C^{4}}^{2}+\|f(\mu)\|_{C^{2}}^{2}+\left\|f^{\prime}(\mu)\right\|_{C^{2}}^{2}$.

Fixing $\xi_{1}$ and $\xi_{2}$ such that $\frac{d_{0}}{2}-6 \xi_{1}^{2}>0, \frac{d_{0}}{2}-6 \xi_{2}^{2}>0$, it follows that there exist positive constants $C_{i}, i=1,2$, such that

$$
\begin{aligned}
& \left\|e_{H}^{\prime}(t)\right\|_{H}^{2}+\int_{0}^{t}\left\|e_{H}^{\prime}(s)\right\|_{H}^{2} d s+\left\|e_{H}(t)\right\|_{H}^{2}+\left\|\nabla_{H} e_{H}(t)\right\|_{H}^{2} \\
& \quad \leq C_{1} H_{\text {max }}^{4}\left(R(t)+\int_{0}^{t} R(s) d s\right)+C_{2} \int_{0}^{t}\left(\left\|\nabla_{H} e_{H}(s)\right\|_{H}^{2}+\left\|e_{H}(s)\right\|_{H}^{2}\right) d s, t \in(0, T] .
\end{aligned}
$$

Applying Gronwall's Lemma to (21) we arrive at (14).

Theorem 1 enables us to conclude that

$$
\left\|e_{H}^{\prime}(t)\right\|_{H}^{2}+\int_{0}^{t}\left\|e_{H}^{\prime}(s)\right\|_{H}^{2} d s+\left\|e_{H}(t)\right\|_{H}^{2}+\left\|\nabla_{H} e_{H}(t)\right\|_{H}^{2} \leq C H_{\text {max }}^{4},
$$

and consequently

$$
\left\|e_{H}^{\prime}(t)\right\|_{H}^{2}+\left\|\nabla_{H} e_{H}(t)\right\|_{H}^{2} \leq C H_{\max }^{4} .
$$

We conclude that the IBVP defined by (11), (9), and (12) or equivalently by (8), (9), and (12) leads to a semi-discrete approximation $u_{H}(t)$ whose kinetic and potential energy are second order approximations for the correspondent quantities of the solution of the IBVP (1)-(3). We remark that the proof of Theorem 1 requires that $u(t), u^{\prime}(t) \in C^{4}(\bar{\Omega})$ and $f(t), f^{\prime}(t) \in C^{2}(\bar{\Omega})$.

\section{Convergence analysis: non-smooth case}

In this section we establish an upper bound analogous to (14) but under weaker assumptions than those used in the proof of Theorem 1, namely $u \in C^{1}\left(0, T, C^{4}(\bar{\Omega})\right) \cap C^{2}(0, T, C(\bar{\Omega}))$ and $f \in C^{1}\left(0, T, C^{2}(\bar{\Omega})\right)$. The main ingredient in the proof of the next result is the Bramble-Hilbert Lemma $([7])$. Let us assume that

$$
u \in \mathcal{V}_{0}=\left\{v \in H^{3}\left(0, T, H^{2}(\Omega)\right) \cap H^{1}\left(0, T, H^{3}(\Omega)\right): v=0 \text { on } \partial \Omega\right\} .
$$

We remark that if $u \in H^{m}\left(0, T, H^{p}(\Omega)\right)$ then $u \in C^{m-1}\left(0, T, H^{p}(\Omega)\right), m \in \mathbb{N}, p \in \mathbb{N}_{0}$. In the following we denote by $\|\cdot\|_{H^{m}\left(H^{p}\right)}$ the usual norm in $H^{m}\left(0, T, H^{p}(\Omega)\right), m, p \in \mathbb{N}_{0}$.

Theorem 2. If the solution $u$ of the IBVP (1)-(3) is in $\mathcal{V}_{0}$ then there exist positive constants $C_{i}, i=1,2$, independent of $u, H$, and $T$ such that for $H \in \Lambda$ with $H_{\max }$ small enough

$$
\begin{aligned}
& \left\|e_{H}^{\prime}(t)\right\|_{H}^{2}+\int_{0}^{t}\left\|e_{H}^{\prime}(s)\right\|_{H}^{2} d s+\left\|\nabla_{H} e_{H}(t)\right\|_{H}^{2} \\
& \quad \leq C_{1} e^{C_{2} t} \sum_{\Delta \in \mathcal{T}_{H}}(\operatorname{diam} \Delta)^{4}\left(\|u\|_{C\left(H^{3}\right)}^{2}+\|u\|_{C^{2}\left(H^{2}\right)}^{2}+\|u\|_{H^{1}\left(H^{3}\right)}^{2}+\|u\|_{H^{3}\left(H^{2}\right)}^{2}\right), t \in[0, T] .
\end{aligned}
$$

Proof: It can be shown that the semi-discrete error $e_{H}(t)$ satisfies

$$
\begin{aligned}
\left(a_{H} e_{H}^{\prime \prime}(t), w_{H}\right)_{H}+\left(b_{H} e_{H}^{\prime}(t), w_{H}\right)_{H}=-\left(\left(D_{H} \nabla_{H} e_{H}(t), \nabla_{H} w_{H}\right)\right)_{H} \\
+T_{1}\left(u(t), w_{H}\right)+T_{2}\left(u(t), w_{H}\right), t \in(0, T], \forall w_{H} \in W_{H, 0}
\end{aligned}
$$


where

$$
T_{1}\left(u(t), w_{H}\right)=\left(\left(D_{H} \nabla_{H}\left(R_{H} u(t)\right), \nabla_{H} w_{H}\right)\right)_{H}-\left(-(\nabla \cdot(D \nabla u(t)))_{H}, w_{H}\right)_{H}
$$

and

$$
T_{2}\left(u(t), w_{H}\right)=\left(\left(a u^{\prime \prime}(t)+b u^{\prime}(t)\right)_{H}-R_{H}\left(a u^{\prime \prime}(t)+b u^{\prime}(t)\right), w_{H}\right)_{H} .
$$

In the definitions of $T_{1}\left(w_{H}\right)$ and $T_{2}\left(w_{H}\right),(g)_{H}$ is given by (10) with $f$ replaced by $g=a u^{\prime \prime}(t)+$ $b u^{\prime}(t)$ or $g=\nabla \cdot(D \nabla u(t))$.

Lemma 5.1 of [16] allows us to conclude the following estimate

$$
\left|T_{1}\left(u(t), w_{H}\right)\right| \leq C\left(\sum_{\Delta \in \mathcal{T}_{H}}(\operatorname{diam} \Delta)^{4}\|u(t)\|_{H^{3}(\Delta)}^{2}\right)^{1 / 2}\left\|\nabla_{H} w_{H}\right\|_{H}, w_{H} \in W_{H, 0} .
$$

Moreover, Lemma 5.7 leads to

$$
\left|T_{2}\left(u(t), w_{H}\right)\right| \leq C\left(\sum_{\Delta \in \mathcal{T}_{H}}(\operatorname{diam} \Delta)^{4}\left(\left\|u^{\prime \prime}(t)\right\|_{H^{2}(\Delta)}^{2}+\left\|u^{\prime}(t)\right\|_{H^{2}(\Delta)}^{2}\right)\right)^{1 / 2}\left\|\nabla_{H} w_{H}\right\|_{H},
$$

for $w_{H} \in W_{H, 0}$, and where $C$ denotes a positive constant which is not necessarily the same one in each appearance.

If we take in $(22) w_{H}=e_{H}^{\prime}(t)$ then we obtain

$$
\begin{aligned}
\left(a_{H} e_{H}^{\prime \prime}(t), e_{H}^{\prime}(t)\right)_{H}+\left(b_{H} e_{H}^{\prime}(t), e_{H}^{\prime}(t)\right)_{H}=-\left(\left(D_{H} \nabla_{H} e_{H}(t), \nabla_{H} e_{H}^{\prime}(t)\right)\right)_{H} & \\
& +T_{1}\left(u(t), e_{H}^{\prime}(t)\right)+T_{2}\left(u(t), e_{H}^{\prime}(t)\right) .
\end{aligned}
$$

As we have

$$
T_{i}\left(u(t), e_{H}^{\prime}(t)\right)=\frac{d}{d t} T_{i}\left(u(t), e_{H}(t)\right)-T_{i}\left(u^{\prime}(t), e_{H}(t)\right), i=1,2,
$$

from (25) we deduce

$$
\begin{aligned}
\left\|\sqrt{a_{H}} e_{H}^{\prime}(t)\right\|_{H}^{2}+2 b_{0} \int_{0}^{t}\left\|e_{H}^{\prime}(s)\right\|_{H}^{2} d s+d_{0}\left\|\nabla_{H} e_{H}(t)\right\|_{H}^{2} \leq 2 T_{1}\left(u(t), e_{H}(t)\right) \\
+2 T_{2}\left(u(t), e_{H}(t)\right)-2 \int_{0}^{t}\left(T_{1}\left(u^{\prime}(s), e_{H}(s)\right)+T_{2}\left(u^{\prime}(s), e_{H}(s)\right)\right) d s .
\end{aligned}
$$

Taking in (26) the upper bounds (23) and (24) we get

$$
\begin{aligned}
& a_{0}\left\|e_{H}^{\prime}(t)\right\|_{H}^{2}+2 b_{0} \int_{0}^{t}\left\|e_{H}^{\prime}(s)\right\|_{H}^{2} d s+\left(d_{0}-4 \xi_{1}^{2}\right)\left\|\nabla_{H} e_{H}(t)\right\|_{H}^{2} \\
& \leq \frac{1}{2 \xi_{1}^{2}} C \sum_{\Delta \in \mathcal{T}_{H}}(\operatorname{diam} \Delta)^{4}\left(\|u(t)\|_{H^{3}(\Delta)}^{2}+\sum_{\ell=1}^{2}\left\|u^{(\ell)}(t)\right\|_{H^{2}(\Delta)}^{2}\right) \\
&+\frac{1}{2 \xi_{2}^{2}} C \sum_{\Delta \in \mathcal{T}_{H}}(\operatorname{diam} \Delta)^{4} \int_{0}^{t}\left(\left\|u^{\prime}(s)\right\|_{H^{3}(\Delta)}^{2}+\sum_{\ell=2}^{3}\left\|u^{(\ell)}(s)\right\|_{H^{2}(\Delta)}^{2}\right) d s \\
&+4 \xi_{2}^{2} \int_{0}^{t}\left\|\nabla_{H} e_{H}(s)\right\|_{H}^{2} d s,
\end{aligned}
$$

where $\xi_{i}, i=1,2$, are non-zero constants. We finish the proof fixing $\xi_{1}$ such that $d_{0}-4 \xi_{1}^{2}>0$, and applying Gronwall's Lemma.

Theorem 2 allows us to conclude that

$\left\|e_{H}^{\prime}(t)\right\|_{H}^{2}+\left\|\nabla_{H} e_{H}(t)\right\|_{H}^{2} \leq C H_{\max }^{4}\left(\|u\|_{C\left(H^{3}\right)}^{2}+\|u\|_{C^{2}\left(H^{2}\right)}^{2}+\|u\|_{H^{1}\left(H^{3}\right)}^{2}+\|u\|_{H^{3}\left(H^{2}\right)}^{2}\right), t \in[0, T]$,

under weaker conditions than those imposed in Theorem 1. 


\section{$5 \quad$ Fully discrete approximation in time and space}

Let us introduce in $[0, T]$ the time grid $\left\{t_{n}=n \Delta t, n=0, \ldots, M_{t}\right\}$ with $t_{M_{t}}=T$ and where $\Delta t$ is the uniform time step. Let also $D_{2, t}$ be the second order centered finite difference operator in time and let $D_{-t}$ be the first order backward finite difference operator in time. The fully discrete in time and space approximation for the solution of the IBVP (1)-(3) is defined by

$$
\begin{aligned}
\left(a_{H} D_{2, t} u_{H}^{n}, w_{H}\right)_{H}+\left(b_{H} D_{-t} u_{H}^{n+1}, w_{H}\right)_{H}=-( & \left.\left(D_{H} \nabla_{H} u_{H}^{n+1}, \nabla_{H} w_{H}\right)\right)_{H} \\
& +\left(f_{H}\left(t_{n+1}\right), w_{H}\right)_{H}, n=1, \ldots, M_{t}-1,
\end{aligned}
$$

for $w_{H} \in W_{H, 0}$, with the initial conditions

$$
\left\{\begin{array}{l}
D_{-t} u_{H}^{1}=R_{H} \phi_{1} \\
u_{H}^{0}=R_{H} \phi_{0}
\end{array}\right.
$$

and the boundary condition

$$
u_{H}^{n}=0 \text { on } \partial \Omega_{H}, n=0, \ldots, M_{t} .
$$

Equivalently, equation (27) can be written as

$$
a_{H} D_{2, t} u_{H}^{n}+b_{H} D_{-t} u_{H}^{n+1}=\nabla_{H}^{*} \cdot\left(D_{H} \nabla_{H} u_{H}^{n+1}\right)+f_{H}\left(t_{n+1}\right) \text { in } \Omega_{H}, n=1, \ldots, M_{t}-1 .
$$

The main theorem of this section is stated next.

Theorem 3. If the solution of the IBVP (1)-(3) is in

$$
H^{1}\left(0, T, H^{3}(\Omega) \cap H_{0}^{1}(\Omega)\right) \cap H^{3}\left(0, T, H^{2}(\Omega)\right) \cap C^{3}(0, T, C(\bar{\Omega})) \cap C^{2}\left(0, T, C^{1}(\bar{\Omega})\right),
$$

then, for $H \in \Lambda$ with $H_{\max }$ small enough, there exists a positive constant $C$, independent of $u$ and $H$, such that for the error $e_{H}^{n}=R_{H} u\left(t_{n}\right)-u_{H}^{n}, n=1, \ldots, M_{t}$, holds the following

$$
\begin{aligned}
\left\|D_{-t} e_{H}^{n}\right\|_{H}^{2}+ & \Delta t \sum_{j=1}^{n}\left\|D_{-t} e_{H}^{j}\right\|_{H}^{2}+\left\|\nabla_{H} e_{H}^{n}\right\|_{H}^{2} \\
\leq & C\left(\Delta t^{2}\left(\|u\|_{C^{4}(C)}^{2}+\Delta t^{2}\|u\|_{C^{2}\left(C^{1}\right)}^{2}+H_{m a x}^{2}\|u\|_{C^{1}\left(C^{2}\right)}^{2}\right)\right. \\
& +\sum_{\Delta \in \mathcal{T}_{H}}(\operatorname{diam} \Delta)^{4}\left(\|u\|_{C\left(H^{3}\right)}^{2}+\|u\|_{C^{2}\left(H^{2}\right)}^{2}\right) \\
& \left.+\Delta t \sum_{\Delta \in \mathcal{T}_{H}}(\operatorname{diam} \Delta)^{4}\left(\|u\|_{H^{1}\left(H^{3}\right)}^{2}+\|u\|_{H^{3}\left(H^{2}\right)}^{2}\right)\right) .
\end{aligned}
$$

Proof: It can be shown that the error $e_{H}^{n}$ satisfies the following equation

$$
\begin{array}{r}
\left(a_{H} D_{2, t} e_{H}^{n}, D_{-t} e_{H}^{n+1}\right)_{H}+\left(b_{H} D_{-t} e_{H}^{n+1}, D_{-t} e_{H}^{n+1}\right)_{H}=-\left(\left(D_{H} \nabla_{H} e_{H}^{n+1}, \nabla_{H} D_{-t} e_{H}^{n+1}\right)\right)_{H} \\
+\sum_{\ell=1}^{3} T_{\ell}\left(u\left(t_{n+1}\right), D_{-t} e_{H}^{n+1}\right),
\end{array}
$$

where

$$
T_{1}\left(u\left(t_{n+1}\right), w_{H}\right)=-\left(\left(D_{H} \nabla_{H}\left(R_{H} u\left(t_{n+1}\right)\right), \nabla_{H} w_{H}\right)_{H}+\left(-\left(\nabla \cdot\left(D \nabla u\left(t_{n+1}\right)\right)\right)_{H}, w_{H}\right)_{H}\right.
$$




$$
T_{2}\left(u\left(t_{n+1}\right), w_{H}\right)=\left(\left(a u^{\prime \prime}\left(t_{n+1}\right)+b u^{\prime}\left(t_{n+1}\right)\right)_{H}-R_{H}\left(a u^{\prime \prime}\left(t_{n+1}\right)+b u^{\prime}\left(t_{n+1}\right)\right), w_{H}\right)_{H},
$$

and

$T_{3}\left(u\left(t_{n+1}\right), w_{H}\right)=\left(a_{H}\left(D_{2, t} R_{H} u\left(t_{n+1}\right)-R_{H} u^{\prime \prime}\left(t_{n+1}\right)\right)+b_{H}\left(D_{-t} R_{H} u\left(t_{n+1}\right)-R_{H} u^{\prime}\left(t_{n+1}\right)\right), w_{H}\right)_{H}$, for $w_{H} \in W_{H, 0}$.

As we have

$$
\begin{gathered}
\left(a_{H} D_{2, t} e_{H}^{n}, D_{-t} e_{H}^{n+1}\right)_{H} \geq \frac{1}{2 \Delta t}\left(\left\|\sqrt{a}_{H} D_{-t} e_{H}^{n+1}\right\|_{H}^{2}-\left\|\sqrt{a}_{H} D_{-t} e_{H}^{n}\right\|_{H}^{2}\right) \\
\left(D_{H} \nabla_{H} e_{H}^{n+1}, \nabla_{H} D_{-t} e_{H}^{n+1}\right)_{H} \geq \frac{1}{2 \Delta t}\left(\left\|\sqrt{D_{H}} \nabla_{H} e_{H}^{n+1}\right\|_{H}^{2}-\left\|\sqrt{D_{H}} \nabla_{H} e_{H}^{n}\right\|_{H}^{2}\right)
\end{gathered}
$$

(see [8]), and

$$
T_{\ell}\left(u\left(t_{n+1}\right), D_{-t} e_{H}^{n+1}\right)=D_{-t} T_{\ell}\left(u\left(t_{n+1}\right), e_{H}^{n+1}\right)-T_{\ell}\left(D_{-t} u\left(t_{n+1}\right), e_{H}^{n}\right), \ell=1,2,
$$

we get

$$
\begin{gathered}
\left\|\sqrt{a_{H}} D_{-t} e_{H}^{n+1}\right\|_{H}^{2}+2 \Delta t b_{0}\left\|D_{-t} e_{H}^{n+1}\right\|_{H}^{2}-2 \sum_{\ell=1}^{2} T_{\ell}\left(u\left(t_{n+1}\right), e_{H}^{n+1}\right)+\left\|\sqrt{D_{H}} \nabla_{H} e_{H}^{n+1}\right\|_{H}^{2} \\
\leq\left\|\sqrt{a_{H}} D_{-t} e_{H}^{n}\right\|_{H}^{2}+\left\|\sqrt{D_{H}} \nabla_{H} e_{H}^{n}\right\|_{H}^{2}-2 \sum_{\ell=1}^{2} T_{\ell}\left(u\left(t_{n}\right), e_{H}^{n}\right) \\
-2 \Delta t \sum_{\ell=1}^{2} T_{\ell}\left(D_{-t} u\left(t_{n+1}\right), e_{H}^{n}\right)+2 \Delta t T_{3}\left(u\left(t_{n+1}\right), D_{-t} e_{H}^{n+1}\right)
\end{gathered}
$$

for $n=1, \ldots, M-1$.

Inequality (31) leads to

$$
\begin{gathered}
\left\|\sqrt{a_{H}} D_{-t} e_{H}^{n+1}\right\|_{H}^{2}+2 \Delta t b_{0} \sum_{j=1}^{n+1}\left\|D_{-t} e_{H}^{j}\right\|_{H}^{2}-2 \sum_{\ell=1}^{2} T_{\ell}\left(u\left(t_{n+1}\right), e_{H}^{n+1}\right)+\left\|\sqrt{D_{H}} \nabla_{H} e_{H}^{n+1}\right\|_{H}^{2} \\
\leq\left\|\sqrt{a_{H}} D_{t} e_{H}^{1}\right\|_{H}^{2}+2 \Delta t\left\|D_{-t} e_{H}^{1}\right\|_{H}^{2}+\left\|\sqrt{D_{H}} \nabla_{H} e_{H}^{1}\right\|_{H}^{2}-2 \sum_{\ell=1}^{2} T_{\ell}\left(u\left(t_{1}\right), e_{H}^{1}\right) \\
-2 \Delta t \sum_{j=1}^{n}\left(\sum_{\ell=1}^{2} T_{\ell}\left(D_{-t} u\left(t_{j+1}\right), e_{H}^{j}\right)+T_{3}\left(u\left(t_{j+1}, D_{-t} e_{H}^{j+1}\right)\right)\right)
\end{gathered}
$$

for $n=1, \ldots, M_{t}-1$. The terms $T_{\ell}\left(u\left(t_{n+1}\right), e_{H}^{n+1}\right), \ell=1,2$, satisfy (23) and (24), respectively, with $w_{H}=e_{H}^{n+1}$, and for $T_{\ell}\left(D_{-t} u\left(t_{n+1}\right), e_{H}^{n}\right), \ell=1,2$, we have

$$
\left|T_{1}\left(D_{-t} u\left(t_{n+1}\right), e_{H}^{n}\right)\right| \leq C \frac{1}{\sqrt{\Delta t}}\left(\sum_{\Delta \in \mathcal{T}_{H}}(\operatorname{diam} \Delta)^{4}\|u\|_{H^{1}\left(t_{n}, t_{n+1}, H^{3}(\Delta)\right)}^{2}\right)^{1 / 2}\left\|\nabla_{H} e_{H}^{n}\right\|_{H}
$$

and

$$
\left|T_{2}\left(D_{-t} u\left(t_{n+1}\right), e_{H}^{n}\right)\right| \leq C \frac{1}{\sqrt{\Delta t}}\left(\sum_{\Delta \in \mathcal{T}_{H}}(\operatorname{diam} \Delta)^{4}\|u\|_{H^{3}\left(t_{n}, t_{n+1}, H^{2}(\Delta)\right)}^{2}\right)^{1 / 2}\left\|\nabla_{H} e_{H}^{n}\right\|_{H} .
$$


For the term $T_{3}\left(u\left(t_{n+1}\right), D_{-t} e_{H}^{n+1}\right)$ it is easy to show the following

$$
\left|T_{3}\left(u\left(t_{n+1}\right), D_{-t} e_{H}^{n+1}\right)\right| \leq C \Delta t\|u\|_{C^{3}(C)}\left\|D_{-t} e_{H}^{n+1}\right\|_{H} .
$$

Considering in (31) the upper bounds (23), (24) for $\left|T_{\ell}\left(u\left(t_{n+1}\right), e_{H}^{n+1}\right)\right|, \ell=1,2$, and (32), (33) for $\left|T_{\ell}\left(D_{-t} u\left(t_{n+1}\right), e_{H}^{n}\right)\right|, \ell=1,2$, and (34) for $\left|T_{3}\left(u\left(t_{n+1}\right), D_{-t} e_{H}^{n+1}\right)\right|$, we find

$$
\begin{aligned}
& a_{0}\left\|D_{-t} e_{H}^{n+1}\right\|_{H}^{2}+2 \Delta t\left(b_{0}-\xi_{3}^{2}\right) \sum_{j=1}^{n+1}\left\|D_{-t} e_{H}^{j}\right\|_{H}^{2}+\left(d_{0}-4 \xi_{1}^{2}\right)\left\|\nabla_{H} e_{H}^{n+1}\right\|_{H}^{2} \\
& \leq\left\|\sqrt{a_{H}} D_{-t} e_{H}^{1}\right\|_{H}^{2}+2 \Delta t b_{0}\left\|D_{-t} e_{H}^{1}\right\|_{H}^{2}+\left\|\sqrt{D_{H}} \nabla_{H} e_{H}^{1}\right\|_{H}^{2}+T_{n+1}(u)+\Delta t \sum_{j=1}^{n} 4 \xi_{2}^{2}\left\|\nabla_{H} e_{H}^{j}\right\|_{H}^{2}
\end{aligned}
$$

for $n=1, \ldots, M_{t}-1$, and where

$$
\begin{aligned}
T_{n+1}(u)= & C\left(\frac{1}{2 \xi_{1}^{2}} \sum_{\Delta \in \mathcal{T}_{H}}(\operatorname{diam} \Delta)^{4}\left(\left\|u\left(t_{n+1}\right)\right\|_{H^{3}(\Delta)}^{2}+\sum_{\ell=1}^{2}\left\|u^{(\ell)}\left(t_{n+1}\right)\right\|_{H^{2}(\Delta)}^{2}\right)\right. \\
& +C \Delta t \sum_{j=0}^{n}\left(\frac{1}{2 \xi_{2}^{2}} \sum_{\Delta \in \mathcal{T}_{H}}(\operatorname{diam} \Delta)^{4}\left(\|u\|_{H^{1}\left(t_{j}, t_{j+1}, H^{3}(\Delta)\right)}^{2}+\|u\|_{H^{3}\left(t_{j}, t_{j+1}, H^{2}(\Delta)\right)}^{2}\right)\right. \\
& \left.+\frac{1}{2 \xi_{3}^{2}} \Delta t^{2}\|u\|_{C^{3}\left(t_{j}, t_{j+1}, C(\bar{\Omega})\right)}^{2}\right)
\end{aligned}
$$

with $\xi_{i}, i=1,2,3$, non-zero constants.

Fixing $\xi_{1}$ and $\xi_{3}$ such that $d_{0}-4 \xi_{1}^{2}>0, b_{0}-\xi_{3}^{2}>0$, and considering the discrete Gronwall's Lemma we conclude that there exist positive constant $C_{1}, C_{2}$ such that

$$
\begin{aligned}
& \left\|D_{-t} e_{H}^{n+1}\right\|_{H}^{2}+\Delta t \sum_{j=1}^{n+1}\left\|D_{-t} e_{H}^{j}\right\|_{H}^{2}+\left\|\nabla_{H} e_{H}^{n+1}\right\|_{H}^{2} \\
& \quad \leq C_{1}\left(\left\|D_{-t} e_{H}^{1}\right\|_{H}^{2}+\left\|\nabla_{H} e_{H}^{1}\right\|_{H}^{2}+\max _{j=2, \ldots, n+1} T_{j}(u)\right)\left(1+T e^{C_{2} n \Delta t}\right),
\end{aligned}
$$

for $n=1, \ldots, M_{t}-1$. To obtain the final error estimate we need to compute an upper bound for $\left\|D_{-t} e_{H}^{1}\right\|_{H}^{2}$ and $\left\|\nabla_{H} e_{H}^{1}\right\|_{H}^{2}$. From the first relation of (28) and as the initial velocity is defined by $\phi_{1}$, we have

$$
D_{-t} e_{H}^{1}=D_{-t} R_{H} u\left(t_{1}\right)-R_{H} u^{\prime}\left(t_{0}\right)
$$

and then

$$
\left\|D_{-t} e_{H}^{1}\right\|_{H}^{2} \leq \frac{1}{2} \Delta t^{2}\|u\|_{C^{2}(C)}^{2}
$$

We also have

$$
\nabla_{H} D_{-t} e_{H}^{1}=\nabla_{H} D_{-t} R_{H} u\left(t_{1}\right)-R_{H} \nabla u^{\prime}(t)+O\left(H_{\max }\right),
$$

where $\left|O\left(H_{\max }\right)\right| \leq C H_{\max }\|u\|_{C^{1}\left(C^{2}\right)}$. Since $\nabla_{H} D_{-t} R_{H} u\left(t_{1}\right)-R_{H} \nabla u^{\prime}(t)=O\left(\Delta t+H_{\max }\right)$, where $\left|O\left(\Delta t+H_{\max }\right)\right| \leq C\left(H_{\max }\|u\|_{C^{1}\left(C^{2}\right)}+\Delta t\|u\|_{C^{2}\left(C^{1}\right)}\right)$, then

$$
\left(\left(\nabla_{H} D_{-t} e_{H}^{1}, \nabla_{H} e_{H}^{1}\right)\right)_{H}=\left(\left(T\left(u\left(t_{1}\right)\right), \nabla_{H} e_{H}^{1}\right)\right)_{H},
$$

with

$$
\left|T\left(u\left(t_{1}\right)\right)\right| \leq C\left(\Delta t\|u\|_{C^{2}\left(C^{1}\right)}+H_{\max }\|u\|_{C^{1}\left(C^{2}\right)}\right) .
$$


From (37) we obtain

$$
\left(1-2 \xi^{2}\right)\left\|\nabla_{H} e_{H}^{1}\right\|_{H}^{2} \leq\left\|\nabla_{H} e_{H}^{0}\right\|_{H}^{2}+\frac{\Delta t^{2}}{2 \xi^{2}}\left\|T\left(u\left(t_{1}\right)\right)\right\|_{H}^{2},
$$

where $\xi \neq 0$. As $e_{H}^{0}=0$, then, there exists a positive constant $C$ such that

$$
\left\|\nabla_{H} e_{H}^{1}\right\|_{H}^{2} \leq C \Delta t^{2}\left(\Delta t^{2}\|u\|_{C^{2}\left(C^{1}\right)}+H_{\max }^{2}\|u\|_{C^{1}\left(C^{2}\right)}\right) .
$$

To conclude (30) we observe that combining (36), (38) with (35) we obtain

$$
\begin{aligned}
& \left\|D_{-t} e_{H}^{n+1}\right\|_{H}^{2}+2 \Delta t \sum_{j=1}^{n+1}\left\|D_{-t} e_{H}^{j}\right\|_{H}^{2}+\left\|\nabla_{H} e_{H}^{n+1}\right\|_{H}^{2}+\left\|e_{H}^{n+1}\right\|_{H}^{2} \\
& \quad \leq C\left(\Delta t^{2}\left(\|u\|_{C^{3}(C)}^{2}+\Delta t^{2}\|u\|_{C^{2}\left(C^{1}\right)}^{2}+H_{\max }^{2}\|u\|_{C^{1}\left(C^{2}\right)}^{2}\right)+\max _{\ell=2, \ldots, n+1} T_{\ell}(u)\right),
\end{aligned}
$$

for $n=1, \ldots, M_{t}-1$, and some positive constant $C$.

Theorem 3 allows us to conclude that the numerical scheme defined by (27) or (29), together with the initial conditions (28) and homogeneous Dirichlet boundary conditions, satisfies

$$
\left\|D_{-t} e_{H}^{n}\right\|_{H}^{2}+\left\|\nabla_{H} e_{H}^{n}\right\|_{H}^{2} \leq C\left(\Delta t^{2}+H_{\max }^{4}\right) .
$$

The first order term in the previous upper bound is due to the discretization of the first order time derivatives in the wave equation and in the initial condition using a first order operator. To increase the time convergence order we should increase the order of the time discretization. We rewrite the IBVP (1), (2) and (3) with $f=0$ in the equivalent form

$$
\left\{\begin{array}{l}
a \frac{\partial u}{\partial t}=w-b u \\
\frac{\partial w}{\partial t}=\nabla \cdot(D \nabla u) \quad \text { in } \Omega \times(0, T]
\end{array}\right.
$$

with the initial conditions

$$
\left\{\begin{array}{l}
w(x, y, 0)=a \phi_{1}(x, y)+b \phi_{0}(x, y) \\
u(x, y, 0)=\phi_{0}(x, y), \quad(x, y) \in \Omega
\end{array}\right.
$$

and the boundary conditions

$$
\left\{\begin{array}{l}
w(x, y, t)=0 \\
u(x, y, t)=0 \quad(x, t) \in \partial \Omega \times(0, T] .
\end{array}\right.
$$

To get a second order approximation for $u$ and $w$ we use a standard procedure used in first order time derivative problems: we consider the Crank-Nicolson approach. Let $u_{H}^{n}$ and $w_{H}^{n}$ be the corresponding approximations defined by the finite difference scheme: for $n=0, \ldots, M_{t}-1$,

$$
\begin{cases}a_{H} D_{-t} u_{H}^{n+1}=\frac{w_{H}^{n+1}+w_{H}^{n}}{2}-b_{H} \frac{u_{H}^{n+1}+u_{H}^{n}}{2} & \\ D_{-t} w_{H}^{n+1}=\nabla \cdot\left(D_{H} \nabla_{H}\left(\frac{u_{H}^{n+1}+u_{H}^{n}}{2}\right)\right) & \text { in } \Omega_{H},\end{cases}
$$




$$
\left\{\begin{array}{l}
w_{H}^{0}=a_{H} R_{H} \phi_{1}+b_{H} R_{H} \phi_{0} \\
u_{H}^{0}=R_{H} \phi_{0} \quad \text { in } \Omega_{H}
\end{array}\right.
$$

and

$$
\left\{\begin{array}{l}
w_{H}^{n}=0 \\
u_{H}^{n}=0 \quad \text { on } \partial \Omega \times\left\{1, \ldots, M_{t}\right\} .
\end{array}\right.
$$

We observe that the previous solutions $u_{H}^{n}$ and $w_{H}^{n}$ satisfy

$$
\left\{\begin{array}{l}
\left(a_{H} D_{-t} u_{H}^{n+1}, v_{H}\right)_{H}=\left(\frac{w_{H}^{n+1}+w_{H}^{n}}{2}, v_{H}\right)_{H}-\left(b_{H} \frac{u_{H}^{n+1}+u_{H}^{n}}{2}, v_{H}\right)_{H} \\
\left(D_{-t} w_{H}^{n+1}, v_{H}\right)_{H}=-\left(\left(D_{H} \nabla_{H}\left(\frac{u_{H}^{n+1}+u_{H}^{n}}{2}\right), \nabla_{H} v_{H}\right)\right)_{H} \quad \forall v_{H} \in W_{H, 0} .
\end{array}\right.
$$

We remark that we also have

$$
\begin{aligned}
\left(\left(a_{H} D_{-t} \nabla_{H} u_{H}^{n+1}, \nabla_{H} v_{H}\right)\right)_{H} & =\left(\left(\nabla_{H}\left(\left(\frac{w_{H}^{n+1}+w_{H}^{n}}{2}\right), \nabla_{H} v_{H}\right)\right)_{H}\right. \\
& -\left(\left(b_{H} \nabla_{H}\left(\frac{u_{H}^{n+1}+u_{H}^{n}}{2}\right), \nabla_{H} v_{H}\right)\right)_{H},
\end{aligned}
$$

for all $v_{H} \in W_{H, 0}$.

By $e_{u}^{n}$ and $e_{w}^{n}$ we represent the global errors in $u_{H}^{n}$ and $w_{H}^{n}$, respectively. It can be shown that

$$
\left(D_{-t} e_{w}^{n+1}, v_{h}\right)_{H}=-\left(\left(D_{H} \nabla_{H}\left(\frac{e_{u}^{n+1}+e_{u}^{n}}{2}\right), \nabla_{H} v_{H}\right)\right)_{H}+\left(T_{1, H}^{n}(u), v_{H}\right)_{H},
$$

for all $v_{H} \in W_{H, 0}$, where

$$
\begin{aligned}
\left\|T_{1, H}^{n}(u)\right\|_{H} \leq C & \left(\Delta t^{4}\|u\|_{C^{4}(C)}+\left(\sum_{\Delta \in \mathcal{T}_{H}}(\operatorname{diam} \Delta)^{4}\left(\left\|u\left(t_{n+1}\right)\right\|_{H^{3}(\Delta)}^{2}+\left\|u\left(t_{n}\right)\right\|_{H^{3}(\Delta)}^{2}\right)\right)^{1 / 2}\right. \\
& \left.+\left(\sum_{\Delta \in \mathcal{T}_{H}}(\operatorname{diam} \Delta)^{4}\left(\left\|\frac{\partial w}{\partial t}\left(t_{n+1}\right)\right\|_{H^{2}(\Delta)}^{2}+\left\|\frac{\partial w}{\partial t}\left(t_{n}\right)\right\|_{H^{2}(\Delta)}^{2}\right)\right)^{1 / 2}\right) \\
\leq C & \left(\Delta t^{4}\|u\|_{C^{4}(C)}+\left(\sum_{\Delta \in \mathcal{T}_{H}}(\operatorname{diam} \Delta)^{4}\|u\|_{C\left(H^{3}\right)}^{2}\right)^{1 / 2}\right. \\
& \left.+\left(\sum_{\Delta \in \mathcal{T}_{H}}(\operatorname{diam} \Delta)^{4}\|u\|_{C^{2}\left(H^{2}\right)}^{2}\right)^{1 / 2}\right) .
\end{aligned}
$$

Taking $v_{H}=e_{w}^{n+1}+e_{w}^{n}$ we obtain

$$
\left\|e_{w}^{n+1}\right\|_{H}^{2}=\left\|e_{w}^{n}\right\|_{H}^{2}-\Delta t\left(\left(D_{H} \nabla_{H}\left(\frac{e_{u}^{n+1}+e_{u}^{n}}{2}\right), \nabla_{H}\left(e_{w}^{n+1}+e_{w}^{n}\right)\right)\right)_{H}+\Delta t\left(T_{1}^{n}(u), e_{w}^{n+1}+e_{w}^{n}\right)_{H} .
$$

From (46) we can deduce the following

$$
\begin{aligned}
\left(\left(D_{H} \nabla_{H}\left(\frac{e_{w}^{n+1}+e_{w}^{n}}{2}\right), \nabla_{H}\left(e_{u}^{n+1}+e_{u}^{n}\right)\right)\right)_{H} & =\left(\left(a_{H} D_{H} D_{-t} \nabla_{H} e_{u}^{n+1}, \nabla_{H}\left(e_{u}^{n+1}+e_{u}^{n}\right)\right)\right)_{H} \\
& +\left(\left(b_{H} D_{H} \nabla_{H}\left(\frac{e_{u}^{n+1}+e_{u}^{n}}{2}\right), \nabla_{H}\left(e_{u}^{n+1}+e_{u}^{n}\right)\right)\right)_{H} \\
& +\left(\left(T_{2, H}^{n}(u), \nabla_{H}\left(e_{u}^{n+1}+e_{u}^{n}\right)\right)\right)_{H},
\end{aligned}
$$


where

$$
\begin{aligned}
&\left\|T_{2, H}^{n}(u)\right\|_{H} \leq C \Delta t^{3 / 2}\left(\left(\sum_{i=1}^{N} \sum_{j=1}^{M-1} k_{j+1 / 2} \int_{t_{n}}^{t_{n+1}} \int_{x_{i-1}}^{x_{i}}\left(\frac{\partial^{4} u}{\partial t^{3} \partial x}\left(x, y_{j}, t\right)\right)^{2} d x d t\right)^{1 / 2}\right. \\
&\left.+\left(\sum_{i=1}^{N-1} \sum_{j=1}^{M} h_{i+1 / 2} \int_{t_{n}}^{t_{n+1}} \int_{y_{j-1}}^{y_{j}}\left(\frac{\partial^{4} u}{\partial t^{3} \partial y}\left(x_{i}, y, t\right)\right)^{2} d y d t\right)^{1 / 2}\right) \\
& \leq C \Delta t^{2}\|u\|_{C^{3}\left(C^{1}\right)} .
\end{aligned}
$$

Inserting (50) in (49) we get

$$
\begin{aligned}
\left\|e_{w}^{n+1}\right\|_{H}^{2}+\left\|\sqrt{a_{H} D_{H}} \nabla_{H} e_{u}^{n+1}\right\|_{H}^{2} & =\left\|e_{w}^{n}\right\|_{H}^{2}+\left\|\sqrt{a_{H} D_{H}} \nabla_{H} e_{u}^{n}\right\|_{H}^{2} \\
& +\Delta t\left(T_{1, H}^{n}(u), e_{w}^{n+1}+e_{w}^{n}\right)_{H} \\
& -\Delta t\left(\left(T_{2, H}^{n}(u), \nabla_{H}\left(e_{u}^{n+1}+e_{u}^{n}\right)\right)\right)_{H} .
\end{aligned}
$$

Equality (52) leads to

$$
\begin{aligned}
\left\|e_{w}^{n+1}\right\|_{H}^{2}+\left\|\sqrt{a_{H} D_{H}} \nabla_{H} e_{u}^{n+1}\right\|_{H}^{2} \leq & \frac{1+2 \epsilon^{2} \Delta t}{1-2 \epsilon^{2} \Delta t}\left(\left\|e_{w}^{n}\right\|_{H}^{2}+\left\|\sqrt{a_{H} D_{H}} \nabla_{H} e_{u}^{n}\right\|_{H}^{2}\right) \\
& +\frac{\Delta t}{2 \epsilon^{2}\left(1-2 \epsilon^{2} \Delta t\right)}\left(\left\|T_{1, H}^{n}(u)\right\|_{H}^{2}+\left\|\frac{\sqrt{D_{H}^{-1}}}{a_{H}} T_{2, H}^{n}(u)\right\|_{H}^{2}\right) .
\end{aligned}
$$

where $\epsilon \neq 0$, and provided that $1-2 \epsilon^{2} \Delta t>0$.

As $e_{w}^{0}=e_{u}^{0}=0$, from (53) we finally obtain

$$
\left\|e_{w}^{n+1}\right\|_{H}^{2}+\left\|\sqrt{a_{H} D_{H}} \nabla_{H} e_{u}^{n+1}\right\|_{H}^{2} \leq C e^{\frac{4 \epsilon^{2} n \Delta t}{1-2 \epsilon^{2} \Delta t}} \max _{j=0, \ldots, n}\left(\left\|T_{1, H}^{j}(u)\right\|_{H}^{2}+\left\|T_{2, H}^{j}(u)\right\|_{H}^{2}\right) .
$$

where $C>0$ denotes a constant independent of $\Delta t$ and $H$.

Finally, using the Poincaré-Friedrichs's inequality we conclude that

$$
\left\|e_{w}^{n+1}\right\|_{H}^{2}+\left\|\nabla_{H} e_{u}^{n+1}\right\|_{H}^{2}+\left\|e_{u}^{n+1}\right\|_{H}^{2} \leq C\left(\Delta t^{4}+H_{\max }^{4}\right),
$$

provided that

$$
u \in C^{4}\left(0, T, C(\bar{\Omega}) \cap C^{3}\left(0, T, C^{1}(\bar{\Omega}) \cap C^{2}\left(0, T, H^{2}(\Omega)\right) \cap C\left(0, T, H^{3}(\Omega) \cap H_{0}^{1}(\Omega)\right) .\right.\right.
$$

\section{Numerical results}

In the following we present some numerical tests that illustrate the theoretical results obtained in previous sections. In accordance with the discussion in Sections 4 and 5 we consider two problems of type (1)-(3), one with a smooth solution (Example 1) and the other with a non-smooth solution (Example 2). In both cases we take $T=0.05$ and use the uniform time step $\Delta t=10^{-5}$. Moreover, the coefficient functions of the wave equation (1) are given by: $a(x, y)=x^{2}, b(x, y)=1+x+y, d_{1}(x, y)=2+y^{2}$, and $d_{2}(x, y)=1+x$. These functions satisfy the restrictions formerly imposed. 
Example 1 To illustrate the smooth case convergence rate we define the initial conditions (2) and the function $f$ in (1), such that, the exact solution of problem (1)-(3) is given by

$$
u(x, y, t)=e^{t}(1-x)(1-\cos (4 \pi y)) \sin (x y) .
$$

Note that $u$ fulfills the assumptions of Theorem 1, namely, $u \in C^{1}\left(0, T, C^{4}(\bar{\Omega})\right) \cap C^{2}(0, T, C(\bar{\Omega}))$. It can also be verified that $f \in C^{1}\left(0, T, C^{2}(\bar{\Omega})\right)$.

Example 2 For the non-smooth case we consider the exact solution of problem (1)-(3) given by

$$
u(x, y, t)=e^{t} \sin (x y)(2 x-2)(y-1)|2 y-1|^{1+\alpha}, \quad \alpha \in \mathbb{R} .
$$

The initial conditions and the function $f$ are defined in accordance with (2) and (1), respectively. In this example, $u$ is under the conditions of Theorem 2, when $\alpha>2$. On the other hand, e.g., for $\alpha=1.1$, we have that $u(t) \in H^{2}(\Omega)$ and those conditions are not meet.

The two previous examples are successively solved on grids $H_{k}(k=1, \ldots, 6)$ of increasing size. We start with an initial random mesh $H_{1}$ and the grid $H_{k+1}$ is obtained from the grid $H_{k}$ by inserting new grid points at the midpoints of the grid $H_{k}$. To calculate the numerical rate of convergence we define the error

$$
E_{H}=\max _{n=1, \ldots, M_{t}}\left\|D_{-t} e_{H}^{n}\right\|_{H}^{2}+\left\|\nabla_{H} e_{H}^{n}\right\|_{H}^{2}
$$

and use the relation

$$
\text { rate }=\frac{\log E_{H_{k}}-\log E_{H_{k+1}}}{\log 2}
$$

where $E_{H_{k}}$ denotes the error $E_{H}$ on the grid $H_{k}$.

The results obtained for Example 1 are shown in Table 1 and they confirm the theoretical $O\left(H_{\max }^{2}\right)$ convergence rate of Theorem 1. The data in Table 1 for Example 2 with $\alpha=2.1$

\begin{tabular}{|c|c|c|c|c|c|c|c|c|}
\hline \multicolumn{3}{|c|}{ Example 1} & \multicolumn{3}{|c|}{ Example $2(\alpha=2.1)$} & \multicolumn{3}{|c|}{ Example $2(\alpha=1.1)$} \\
\hline$H_{\max }$ & $E_{H}$ & rate & $H_{\max }$ & $E_{H}$ & rate & $H_{\max }$ & $E_{H}$ & rate \\
\hline $1.301 \mathrm{e}-1$ & $4.578 \mathrm{e}-1$ & - & $1.577 \mathrm{e}-1$ & $3.272 \mathrm{e}-2$ & - & $1.713 \mathrm{e}-1$ & $4.228 \mathrm{e}-2$ & - \\
\hline $6.505 \mathrm{e}-2$ & $1.182 \mathrm{e}-1$ & 1.953 & $7.885 \mathrm{e}-2$ & 8.697e-3 & 1.912 & $8.566 \mathrm{e}-2$ & $2.005 \mathrm{e}-2$ & 1.076 \\
\hline $3.252 \mathrm{e}-2$ & $2.973 \mathrm{e}-2$ & 1.991 & $3.943 \mathrm{e}-2$ & $2.200 \mathrm{e}-3$ & 1.983 & $4.283 \mathrm{e}-2$ & $9.440 \mathrm{e}-3$ & 1.087 \\
\hline $1.626 \mathrm{e}-2$ & $7.441 \mathrm{e}-3$ & 1.998 & $1.971 \mathrm{e}-2$ & $5.513 \mathrm{e}-4$ & 1.997 & $2.141 \mathrm{e}-2$ & $4.466 \mathrm{e}-3$ & 1.080 \\
\hline $8.131 \mathrm{e}-3$ & $1.860 \mathrm{e}-3$ & 2.000 & $9.857 \mathrm{e}-3$ & $1.378 \mathrm{e}-4$ & 2.001 & $1.071 \mathrm{e}-2$ & $1.978 \mathrm{e}-3$ & 1.175 \\
\hline $4.065 \mathrm{e}-3$ & $4.649 \mathrm{e}-4$ & 2.001 & $4.928 \mathrm{e}-3$ & $3.443 e-5$ & 2.000 & $5.354 \mathrm{e}-3$ & $9.152 \mathrm{e}-4$ & 1.112 \\
\hline
\end{tabular}
also verifies the second order convergence rate of the non-smooth case, as proven in Theorem 2 . Again in Table 1, we display the results obtained for Example 2, but now using $\alpha=1.1$, that is, when the solution $u(t)$ belongs to $H^{2}(\Omega)$. The numerical rate of convergence of order $O\left(H_{\max }\right)$

Table 1: Results of the numerical convergence tests.

suggests that at least the restriction $u(t) \in H^{3}(\Omega)$ of Theorem 2 is optimal, in the sense that cannot be weakness without losing the second order rate. We remark that, using the results established in [16] and following the steps of Theorem 2, it can be proved that the rate of convergence is in fact $O\left(H_{\max }\right)$ when $u(t) \in H^{2}(\Omega)$. For illustration, we present in Figure 1 the numerical solution and square of the error $e_{H}^{n}$ for each of the examples considered.

In the next experiment we examine the rate of convergence in time. For that we solve Example 3 for successively smaller time steps $\Delta t$ and using a fixed spatial grid with $H_{\max }=3.913 \mathrm{e}-3$. 

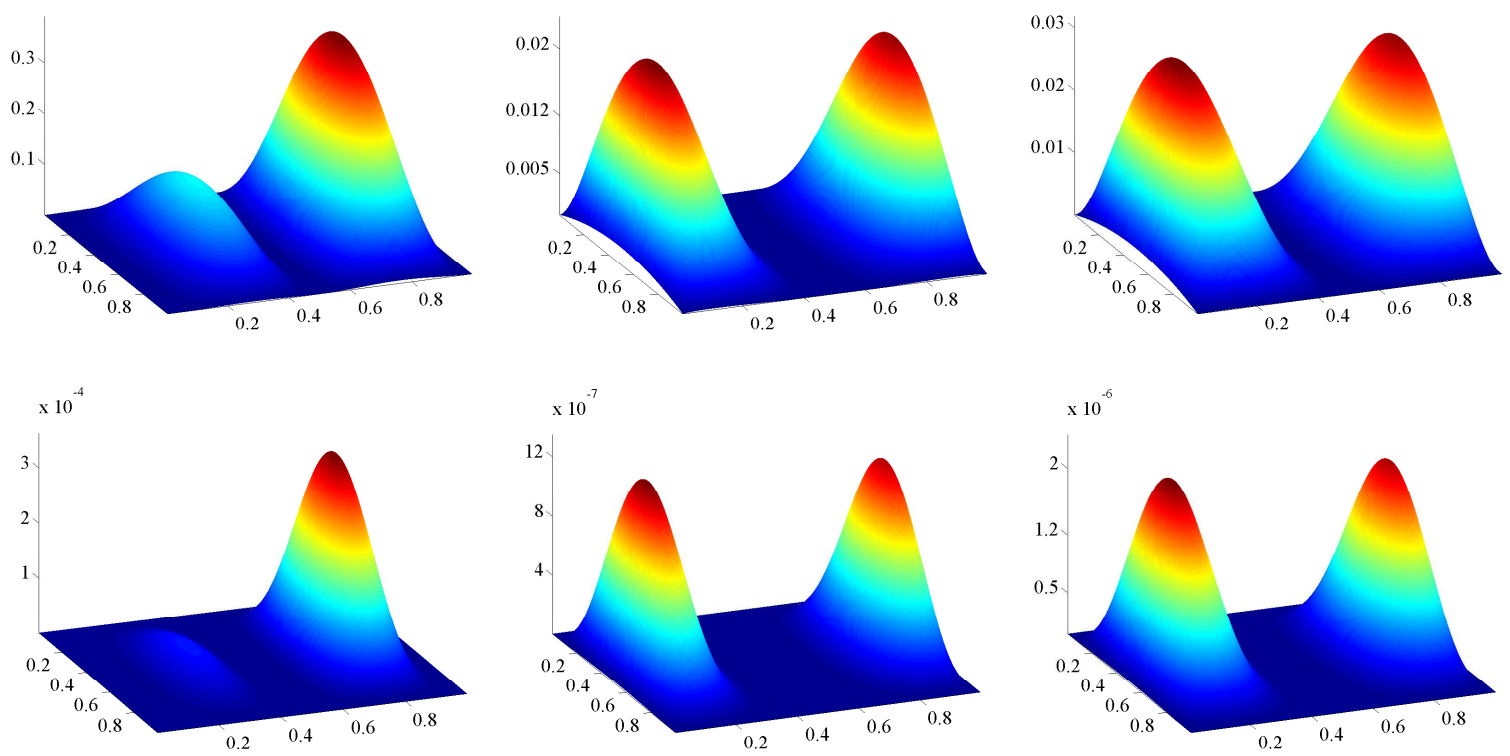

Figure 1: From left to right: numerical solution $u_{H}^{n}$ (first row) and square of the error $e_{H}^{n}$ (second row) on the grid $H_{6}$ at $T=0.05$; for Example 1 and Example 2 with $\alpha=2.1$ and $\alpha=1.1$.

In this example we use a larger value of $T$, namely, $T=3$. The results of our simulation are presented in Table 2 and they confirm the theoretical $O(\Delta t)$ convergence rate of Theorem 3 as well as the $O\left(\Delta t^{2}\right)$ convergence rate of the second order in time scheme (42)-(44). Some representative figures are shown in Figure 2. Note that in accordance with estimate (55) we define the following error expression for the second order scheme

$$
E_{H, 2}=\max _{n=1, \ldots, M_{t}}\left\|e_{w}^{n}\right\|_{H}^{2}+\left\|\nabla_{H} e_{u}^{n}\right\|_{H}^{2}+\left\|e_{u}^{n}\right\|_{H}^{2} .
$$

Example 3 Let us consider problem (1)-(3) with coefficient functions $a(x, y)=5(1+x)$, $b(x, y)=x y, d_{1}(x, y)=1+x$, and $d_{2}(x, y)=y$, exact solution given by

$$
u(x, y, t)=e^{t}(x-1) \sin (\pi x)(y-1) \sin (\pi y),
$$

\begin{tabular}{|c|c|c|c|c|c|}
\hline \multicolumn{3}{|c|}{ Example 3 (2nd order scheme) } & \multicolumn{3}{|c|}{ Example 3 (1st order scheme) } \\
\hline$\Delta t$ & $E_{H, 2}$ & rate & $\Delta t$ & $E_{H}$ & rate \\
\hline $5.000 \mathrm{e}-1$ & $3.950 \mathrm{e}-1$ & - & $5.000 \mathrm{e}-1$ & $24.89 \mathrm{e}-1$ & - \\
\hline $2.500 \mathrm{e}-1$ & $9.949 \mathrm{e}-2$ & 1.989 & $2.500 \mathrm{e}-1$ & $13.78 \mathrm{e}-1$ & 0.853 \\
\hline $1.250 \mathrm{e}-1$ & $2.486 \mathrm{e}-2$ & 2.001 & $1.250 \mathrm{e}-1$ & $7.285 \mathrm{e}-1$ & 0.920 \\
\hline $6.250 \mathrm{e}-2$ & $6.151 \mathrm{e}-3$ & 2.015 & $6.250 \mathrm{e}-2$ & $3.754 \mathrm{e}-1$ & 0.956 \\
\hline $3.125 \mathrm{e}-2$ & $1.478 \mathrm{e}-3$ & 2.057 & $3.125 \mathrm{e}-2$ & $1.907 \mathrm{e}-1$ & 0.977 \\
\hline
\end{tabular}

and properly defined initial conditions and function $f$.

Table 2: Numerical convergence rate in time. 



Figure 2: From left to right: numerical solution $u_{H}^{n}$ obtained with the second order in time scheme and square of the error $e_{u}^{n}$ for the second order in time scheme and the first order in time scheme; results for Example 3 using $\Delta t=0.0625$.

\section{Conclusions}

In this work a numerical scheme for the discretization of a wave type equation was proposed and studied. The main goal was to establish conditions that allow to obtain second order approximations, in space and time, for the kinetic and potential energies with respect to a discrete $L^{2}$-norm.

The main results of this paper are Theorem 1 and Theorem 2. In these theorems convergence properties of the semi-discrete solution defined by the fully discrete in space piecewise linear finite element method (8), (9), and (12), which is equivalent to the finite difference method (11), (9), and (12), were analyzed. Two cases corresponding to smooth and non-smooth assumptions for the solution of the correspondent continuous IBVP were considered. For each case, two complete different techniques of analysis were followed to derive second order approximations for the kinetic and potential energies. Theorem 1 establishes the second order convergence for the smooth case, while Theorem 2 deals with the non-smooth case. The discrete in time version of Theorem 2 was studied in Section 5.

Numerical experiments illustrating the obtained theoretical results were also included. In particular, Example 2 with $\alpha=1.1$, illustrates that the convergence rate established in Theorem 2 is optimal in the sense that if $u(t) \in H^{2}(\Omega)$ then the rate of convergence is only $O\left(H_{\max }\right)$. The proof of this fact can be done following the proof of Theorem 2 and the results presented in $[16]$.

The main motivation for this work is the coupling between the electric potential, or electric field, and diffusion of a drug in a target tissue. In fact, in iontophoresis or electroporation applications, electric fields are used to enhance drug diffusion and absorption by the target tissue. The wave equations governing the electric potential and the electric field, equations (4) and (5), respectively, are particular cases of the general equation (1). In future work we intent to address this more complex problem which is obtained coupling a equation of type (1) with a properly defined parabolic equation for the drug concentration.

\section{Acknowledgments}

This work was partially supported by the Centre for Mathematics of the University of Coimbra - UID/MAT/00324/2013, funded by the Portuguese Government through FCT/MCTES and co-funded by the European Regional Development Fund through the Partnership Agreement PT2020. D. Jordão was supported by FCT PhD scholarships PD/BI/128068/2016 and L. Pinto was also supported by FCT scholarship SFRH/BPD/112687/2015. 


\section{References}

[1] R.M. Alford, K.R. Kelly, D.M. Boore, Accuracy of finite-difference modeling of the acoustic wave equation, Geophysics 39 (1974) 834-842.

[2] M. Baccouch, Superconvergence of the local discontinuous Galerkin method applied to the one-dimensional second-order wave equation, Numer. Meth. Part. D. E. 30 (2014) 862-901.

[3] G.A. Baker, Error estimates for finite element methods for second order hyperbolic equations, SIAM J. Numer. Anal. 13 (1976) 564-576.

[4] S. Barbeiro, J.A. Ferreira, R. Grigorieff, Supraconvergence of a finite difference scheme for solutions in $H^{s}(0, L)$, IMA J. Numer. Anal. 25 (2005) 797-811.

[5] S. Barbeiro, J.A. Ferreira, L. Pinto, $H^{1}$-second order convergent estimates for non-Fickian models, Appl. Numer. Math. 61 (2011) 201-215.

[6] S. Becker, B. Zorec, D. Miklavčič, N. Pavšelj, Transdermal transport pathway creation: Electroporation pulse order, Math. Biosci. 257 (2014) 60-68.

[7] J. Bramble, S. Hilbert, Estimation of linear functionals on Sobolev spaces with application to Fourier transforms and spline interpolation, SIAM J. Numer. Anal. 7 (1970) 112-124.

[8] J. Branco, J.A. Ferreira, A singular perturbation of the heat equation with memory, J. Comput. Appl. Math. 218 (2008) 376-394.

[9] J.H. Brandts, Superconvergence of mixed finite element semi-discretizations of two timedependent problems, Appl. Math-Czech. 44 (1999) 43-53.

[10] J.D. Byrne, M.N.R. Jajja, A.T. O'Neill, L.R. Bickford, A.W. Keeler, N. Hyder, K. Wagner, A. Deal, R.E. Little, R.A. Moffitt, C. Stack, M. Nelson, C.R. Brooks, W. Lee, J.C. Luft, M.E. Napier, D. Darr, C.K. Anders, R. Stack, J.E. Tepper, A.Z. Wang, W.C. Zamboni, J.J. Yeh, J. M. DeSimone, Local iontophoretic administration of cytotoxic therapies to solid tumors, Sci. Transl. Med. 7 (2015) 273ra14.

[11] R. Codina, Finite element approximation of the hyperbolic wave equation in mixed form, Comput. Methods in Appl. Mech. Eng. 197 (2008) 1305-1322.

[12] G. Cohen, P. Joly, Construction analysis of fourth-order finite difference schemes for the acoustic wave equation in nonhomogeneous media, SIAM J. Numer. Anal. 33 (1996) 12661302.

[13] L.C. Cowsat, T.F. Dupont, M.F. Wheeler, A priori estimates for mixed finite element methods for the wave equation, Comput. Methods in Appl. Mech.Eng. 82 (1990) 205-222.

[14] R. Davalosa, B. Rubinskya, L. Mir, Theoretical analysis of the thermal effects during in vivo tissue electroporation, Bioelectrochemistry 61 (2003) 99-107.

[15] T. Dupont, $L^{2}$-estimates for Galerkin methods for second order hyperbolic equations, SIAM J. Numer. Anal. 10 (1973) 880-889.

[16] J.A. Ferreira, R. Grigorieff, Supraconvergence and supercloseness of a scheme for elliptic equations on nonuniform grids, Numer. Func. Anal. Opt. 27 (2006) 539-564. 
[17] J.A. Ferreira, E. Gudiño, P. Oliveira, A second order approximation for quasilinear NonFickian models, Comput. Methods Appl. Math. 13 (2013) 471-493.

[18] J.A. Ferreira, L. Pinto, Supraconvergence and supercloseness in quasilinear coupled problems, J. Comput. Appl. Math. 252 (2013) 120-131.

[19] J.A. Ferreira, L. Pinto, G. Romanazzi, Supraconvergence and supercloseness in Volterra equations, Appl. Numer. Math. 62 (2012) 1718-1739.

[20] Y. Granot, B. Rubinsky, Mass transfer model for drug delivery in tissue cells with reversible electroporation, Int. Heat. Mass Tran. 51 (2008) 5610-5616.

[21] T. Gratieri, Y. Kalia, Mathematical models to describe iontophoretic transport in vitro and in vivo and the effect of current application on the skin barrier, Adv. Drug Deliv. Rev. 65 (2013) 315-329.

[22] T. Jaskari, M. Vuorio, K. Kontturi, A. Urtti, J. Manzanares, J. Hirvonen, Controlled transdermal iontophoresis by ion-exchanges fiber, J. Control. Release 67, 179-190, 2000.

[23] E.W. Jenkins, B. Rivière, M.F. Wheeler, A priori error estimates for mixed finite element approximations of the acoustic wave equation, SIAM J. Numer. Anal. 40 (2002) 1698-1715.

[24] C. Johnson, Discontinuous Galerkin finite element methods for second order hyperbolic problems, Comput. Methods in Appl. Mech. Eng. 107 (1993) 117-129.

[25] J. Larsson, Electromagnetics from a quasistatic perspective, Am. J. Phys. 75 (2007) 230239.

[26] J. Li, S. Shields, Superconvergence analysis of Yee scheme for metamaterial Maxwell's equations on non-uniform rectangular meshes, Numer. Math. 134 (2016) 741-782.

[27] Q. Lin, J. Li, Superconvergence analysis for Maxwell's equations in dispersive media, Math. Comp. 77 (2008) 757-771.

[28] S. Molokhia, Y. Zhang, W. Higuchi, S. Li, Iontophoretic transport across a multiple membrane system, J. Pharm. Sci. 97 (2008), 490-505.

[29] P. Monk, Superconvergence of finite element approximations to Maxwell's equations, Numer. Meth. Part. D. E. 10 (1994) 793-812.

[30] P. Monk, G.R. Richter, A discontinuous Galerkin method for linear symmetric hyperbolic systems in inhomogeneous media, J. Sci. Comput. 22 (2005) 443-477.

[31] P. Monk, E. Süli, A convergence analysis of Yee's scheme on nonuniform grids, SIAM J. Numer. Anal. 31 (1994) 393-412.

[32] M. Patane, A. Cohen, J. Sheppard, Q. Nguyen, Ocular iontophoresis for drug delivery, Retina Today 6 (2011) 64-66.

[33] R. Pignatello, M. Fresta, G. Puglisi, Transdermal drug delivery by iontophoresis. I. fundamentals and theoretical aspects, J. Appl. Cosmetology 14 (1996) 59-72.

[34] B. Rivière, M.F. Wheeler, Discontinuous finite element methods for acoustic and elastic wave problems, Contemp. Math. 329 (2003) 271-282. 
[35] K. Tojo, Mathematical model of iontophoretic transdermal drug delivery, J. Chem. Eng. Jpn. 22 (1989) 512-518.

[36] J. Verwer, J. M. Sanz-Serna, Convergence of method of lines approximations to partial differential equations, Computing 33 (1984) 297-313.

[37] L.B. Wahlbin, Superconvergence in Galerkin Finite Element Methods, Lectures Notes in Mathematics, 1605, Springer, Berlin, 1995.

[38] A. Yadollahpour, Z. Rezaee, Electroporation as a new cancer treatment technique: a review on the mechanisms of action, Biomed. Pharma. J. 7 (2014) 53-62.

[39] A. Zhou, Q. Lin, Optimal superconvergence estimates of finite element method for a scalar hyperbolic equation, Acta Math. Sci. 14 (1994), 90C94. 